\title{
BMJ Open Costs associated with functional gastrointestinal disorders and related signs and symptoms in infants: a systematic review protocol
}

Julie Glanville, ${ }^{1}$ Thomas Ludwig, ${ }^{2}$ Carlos Lifschitz, ${ }^{3}$ James Mahon, ${ }^{1}$ Mohamad Miqdady, ${ }^{4}$ Miguel Saps, ${ }^{5}$ Seng Hock Quak, ${ }^{6}$ Irene Lenoir-Wijnkoop, ${ }^{7}$ Mary Edwards, ${ }^{1}$ Hannah Wood, ${ }^{1}$ Hania Szajewska ${ }^{8}$

To cite: Glanville J, Ludwig T, Lifschitz C, et al. Costs associated with functional gastrointestinal disorders and related signs and symptoms in infants: a systematic review protocol BMJ Open 2016;6:e011475. doi:10.1136/bmjopen-2016011475

- Prepublication history for this paper is available online. To view these files please visit the journal online (http://dx.doi.org/10.1136/ bmjopen-2016-011475).

$\mathrm{JG}$ and $\mathrm{TL}$ contributed equally.

Received 11 February 2016 Revised 8 July 2016 Accepted 29 July 2016

CrossMark

For numbered affiliations see end of article.

Correspondence to Professor Hania Szajewska; hania@ipgate.pl

\section{ABSTRACT}

Introduction: Functional gastrointestinal disorders (FGIDs) and FGID-related signs and symptoms have a fundamental impact on the psychosocial, physical and mental well-being of infants and their parents alike. Recent reviews and studies have indicated that FGIDs and related signs and symptoms may also have a substantial impact on the budgets of third-party payers and/or parents. The objective of this systematic review is to investigate these costs.

Methods and analysis: The population of interest is healthy term infants (under 12 months of age) with colic, regurgitation and/or functional constipation. Outcomes of interest will include the frequency and volume of reported treatments, the cost to third-party payers and/or parents for prescribed or over the counter treatments, visits to health professionals and changes in infant formula purchases, and the loss of income through time taken off work and out of pocket costs. Relevant studies will be identified by searching databases from 2005 onwards (including MEDLINE, EMBASE, PsycINFO, NEXIS, DARE, Health Technology Assessment database, National Health Service Economic Evaluation Database and others), conferences from the previous 3 years and scanning reference lists of eligible studies. Study selection, data extraction and quality assessment will be conducted by two independent reviewers and disagreements resolved in discussion with a third reviewer. Quality assessment will involve study design-specific checklists. Relevant studies will be summarised narratively and presented in tables. An overview of treatments and costs will be provided, with any geographical or other differences highlighted. An assessment of how the totals for cost differ across countries and elements that contribute to the differences will be generated.

Ethics and dissemination: This is a systematic review of published studies that will be submitted for publication to a peer-reviewed journal. Ethical committee approval is not required.

Trial registration number: CRD42016033119.

\section{INTRODUCTION}

Functional gastrointestinal disorders (FGIDs) and related signs and symptoms can have

\section{Strengths and limitations of this study}

- This is a systematic review with a registered protocol, following rigorous methods, including an extensive search, and data extraction and study quality assessment by two independent reviewers.

- The review seeks to identify direct and indirect costs from relevant populations: both studies with healthy term infants under 12 months of age who meet the Rome III criteria for functional constipation, infantile colic and regurgitation and studies of infants of the same age with gastrointestinal symptoms provided that these are linked to one of the aforementioned functional gastrointestinal disorders.

- The review is focused on more recent studies to ensure that currency and most recent practice are reflected in terms of care of functional gastrointestinal disorders and related signs and symptoms.

- The searches are limited to studies published since 2005 in English.

fundamental short-term and long-term impacts on the physical and mental well-being of children and parents. ${ }^{1-6}$ FGIDs include a variable combination of signs and symptoms which cannot be explained by obvious structural or biochemical abnormalities. ${ }^{7-9}$

The literature suggests that more than $50 \%$ of all infants display at least one FGID or related sign and symptom during the first year after birth. ${ }^{10-12}$ A recent meta-review reported that the worldwide prevalence of the three most common FGIDs in infants, infantile regurgitation, colic and functional constipation, is $\sim 30 \%, 20 \%$ and $15 \%$, respectively. ${ }^{6}$

Experts broadly agree that the preferred treatment for infantile colic and regurgitation should be parental reassurance and nutritional advice. ${ }^{13-17}$ Nutritional management of regurgitation involves correcting the frequency and 
volume of feeds and, in some cases of formula-fed infants, the use of antiregurgitation formulas. ${ }^{9}{ }^{18-21}$ Thickened antiregurgitation formulas have been shown to reduce the volume and frequency of regurgitation and crying, to improve sleep and to support weight gain. ${ }^{18-21}$

There is broad consensus in reviews that there is no indication for pharmacological treatment, such as proton pump inhibitors (PPIs) or prokinetic drugs, in infants with infantile colic or regurgitation, even if these are showing signs of distress. These reviews suggest that there is either no evidence of the effectiveness of pharmacological treatment or evidence of no effect, and there can be significant risks and negative side effects. ${ }^{14} 182022-25$

A recent survey of Italian general paediatricians on the implementation of the 2009 North American Society for Pediatric Gastroenterology, Hepatology, and Nutrition (NASPGHAN)-European Society for Pediatric Gastroenterology, Hepatology, and Nutrition (ESPGHAN) guidelines in children with GER symptoms ${ }^{26}$ found that only $2 \%$ of participating paediatricians completely adhered to these guidelines. The study also found that $56 \%$ of paediatricians prescribed PPIs for infants with unexplained crying and/or distressed behaviour and 38\% prescribed PPIs in infants with uncomplicated recurrent regurgitation and vomiting. Of the participating paediatricians, $79 \%$ were reported to overprescribe PPIs.

The Avon Longitudinal Study of Parents and Children (ALSPAC) found that $16 \%$ of infants with colic and flatulence were given activated dimethicone, and $13 \%$ were given gripe water. ${ }^{27}$ Systematic reviews (SRs) have found both remedies to be not effective in the treatment of either of these conditions. ${ }^{1728}$ The use of other medicinal and herbal products for the treatment of infantile colic is also common. Most of these products are either not licensed for use in this age group or their safety and tolerance have not been tested in clinical studies. ${ }^{14} 27-33$

Although some of these FGIDs or related signs and symptoms may not require treatment, besides reassuring and educating parents, parents frequently seek medical attention or treatment. ${ }^{6} 1134$ Infantile regurgitation, colic and constipation in infants are among the most common reasons for parents to consult a healthcare professional in the first months after birth. ${ }^{10}{ }^{11}$ As such, the annual costs for sleeping and crying disorders in the UK are estimated to be in the range of $£ 65$ million. ${ }^{35}$ Infants have the highest rate of emergency department visits for constipation in the $\mathrm{USA}^{36}$ and the average costs of care per patient are US\$2306. ${ }^{36}$

In summary, reviews and studies have indicated a high prevalence of FGIDs and related signs and symptoms in infants, and that these can cause relevant direct and indirect costs that may have a substantial impact on third-party payers and/or parents.

\section{OBJECTIVES}

The primary objective is to conduct an SR of the direct and indirect costs to third-party payers and/or parents of infants with FGIDs and related signs and symptoms.
The SR will seek data to answer the following questions:

- What is the international, national and regional cost of illness (COI) for FGIDs and related signs and symptoms reported in published studies?

- What are the costs of treating FGIDs and related signs and symptoms from the perspective of public payers, health insurers and parents of infants with FGIDs and related signs and symptoms (private purchase of remedies and interventions)?

- What are the indirect costs of FGIDs and related signs and symptoms in terms of parents' days lost from work/reduced productivity and from parental out of pocket expenses?

Using the evidence from the SR, at least one de novo exemplar COI calculation will be undertaken to estimate the total costs associated with the care of infants with FGIDs and related signs and symptoms in a developed economy.

The review protocol has been registered with PROSPERO (CRD42016033119).

\section{METHODS AND ANALYSIS}

The SR eligibility criteria are described below and are summarised in table 1.

\section{Participants}

The population of interest is healthy term infants (under 12 months of age) with colic, regurgitation and/ or functional constipation.

Studies that include infants who meet the Rome III criteria for the clinical diagnosis of infantile colic, regurgitation and/or constipation will be eligible for inclusion. Ideally, the cost of each of these conditions should be explored separately, but it is anticipated that this may not be possible due to the overlap in these conditions. FGID-related signs and symptoms that do not fulfil the Rome criteria will also generate direct and indirect costs (figure 1). Therefore, studies which include infants with symptoms, including hard stools or straining, vomiting, problematic feeding (eg, spitting) or inconsolable or persistent crying, will be eligible for inclusion, provided that these symptoms are linked to at least a suspicion of FGID in all cases (figure 2). Studies with these symptoms where no statement on the suspected underlying cause is made or where the symptoms could be the result of a non-FGID cause will not be eligible for inclusion (figure 1).

Studies involving exclusively preterm infants or where the majority of the infants are preterm will not be eligible.

In all cases, the definition used by authors for FGIDs and related signs and symptoms will be recorded.

Studies of participants from any country are eligible.

\section{Interventions}

Studies reporting on the costs of any intervention or no intervention are eligible for inclusion. 
Table 1 Summary of inclusion/exclusion criteria

\begin{tabular}{|c|c|c|}
\hline Criterion & Inclusion criteria & Exclusion criteria \\
\hline Participants & $\begin{array}{l}\text { Healthy term infants under } 12 \text { months who meet the } \\
\text { Rome III criteria for } \\
\text { Constipation } \\
\text { Infantile colic } \\
\text { Segurgitation } \\
\text { Studies which include infants with symptoms, including } \\
\text { hard stools or straining, vomiting, problematic feeding } \\
\text { (eg, spitting) or inconsolable or persistent crying, will be } \\
\text { eligible for inclusion, provided that these symptoms are } \\
\text { linked to at least a suspicion of FGID in all cases }\end{array}$ & $\begin{array}{l}\text { Infants without FGID. } \\
\text { Infants with vomiting, problematic feeding, } \\
\text { inconsolable or persistent crying and where no } \\
\text { statement on the believed underlying gastrointestinal } \\
\text { cause is made or where the symptoms could be the } \\
\text { result of a non-FGID cause } \\
\text { Preterm infants only or studies where preterm } \\
\text { infants form }>50 \% \text { of the participants }\end{array}$ \\
\hline Interventions & $\begin{array}{l}\text { Any intervention (or no intervention for burden on } \\
\text { parents or carers) }\end{array}$ & \\
\hline Comparators & No comparator is necessary & \\
\hline Outcomes & $\begin{array}{l}\text { Reported treatments of FGIDs and related signs and } \\
\text { symptoms (regardless of effectiveness) and the } \\
\text { frequency and volume of use } \\
\text { Costs to patients and third-party payers of } \\
\text { Prescribed treatments } \\
\text { Over the counter or home remedies } \\
\text { Visits to orthodox and complementary health } \\
\text { professionals and other providers of care } \\
\text { Loss of income for parents/carers from infant FGIDs } \\
\text { and related signs and symptoms through: } \\
\text { Inability to return to work } \\
\text { Time taken off work } \\
\text { Out of pocket costs }\end{array}$ & \\
\hline Study types & $\begin{array}{l}\text { RCTs } \\
\text { Non-randomised controlled trials } \\
\text { Cost of illness studies } \\
\text { Economic evaluations } \\
\text { Observational studies }\end{array}$ & $\begin{array}{l}\text { Non-systematic reviews } \\
\text { Conference abstracts } \\
\text { Letters } \\
\text { Comments } \\
\text { Editorial } \\
\text { News stories } \\
\text { Case reports }\end{array}$ \\
\hline Limits & $\begin{array}{l}\text { Studies published from } 2005 \text { onwards } \\
\text { Studies in English } \\
\text { Full publications }\end{array}$ & $\begin{array}{l}\text { Studies published before } 2005 \\
\text { Studies in languages other than English }\end{array}$ \\
\hline
\end{tabular}

\section{Comparators}

A study with any or no comparator will be eligible for inclusion in this SR.

\section{Outcomes}

The outcomes of interest are:

- Reported treatments of FGIDs and related signs and symptoms (regardless of their effectiveness), or the specific symptom combinations described in the Population description above, and the frequency and volume of use.

- Costs to third-party payers and/or parents of infants with FGIDs and related signs and symptoms of:

- prescribed treatments;

- over the counter or home remedies;

- visits to orthodox and complementary health professionals and other providers of care;

- change in infant formula purchases.

- Loss of income for parents/carers from infants with FGIDs and related signs and symptoms, or the specific symptom combinations described in the Population section above, through:

- inability to return to work;

- time taken off work;

- out of pocket costs.

\section{Study types}

Studies of the following designs will be eligible for inclusion in the review:

- randomised controlled trials (RCTs);

- non-RCTs;

- COI studies;

- economic evaluations;

- observational studies.

Previously published SRs will not be eligible for inclusion, but their reference lists will be checked to identify any additional relevant studies.

Studies published as abstracts or conference presentations will not be included in the review but will be listed for information and used as clues to full publications. 


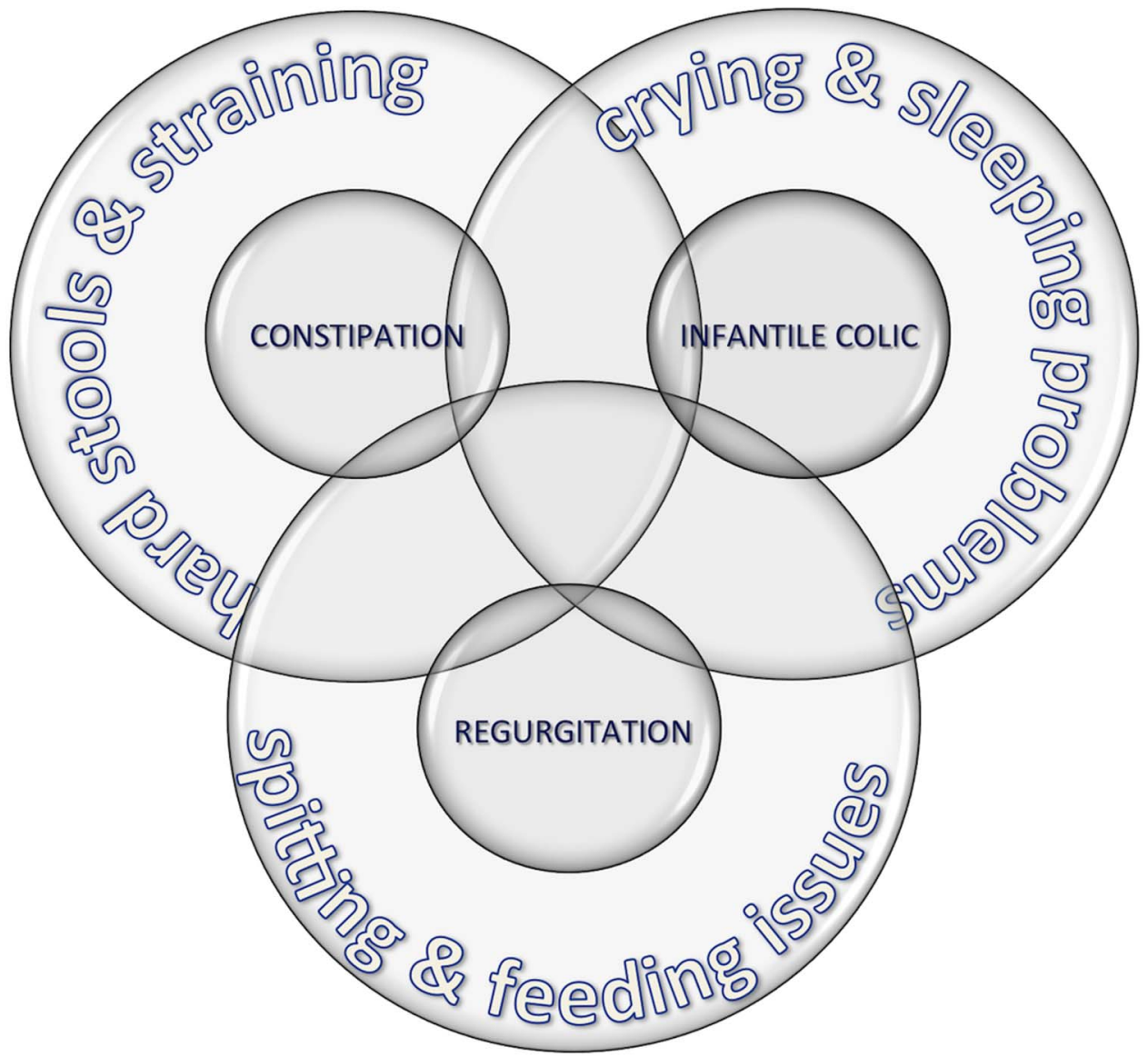

Figure 1 Defining the population of interest. Infants who present in daily paediatric practice or receive treatment for functional gastrointestinal symptoms may not always fulfil the stringent criteria for a functional gastrointestinal disorder (FGID) as defined by the Rome criteria (dark centre). However, these FGID-related symptoms do still cause direct and indirect costs and need to be considered (light circles).

Any study of a design not listed above will not be eligible for inclusion in the SR. This includes, but is not limited to, case studies and non-SRs. Opinion pieces, letters and editorials will not be eligible for inclusion.

\section{Limits}

The searches are limited to English language studies published since 2005 to focus on the most current costs and most recent practice in terms of care of FGIDs and related signs and symptoms.

\section{Identifying relevant studies}

The search strategy to identify studies for this review is shown in figure 2. The search excludes publication types that are unlikely to contain research results. Records of publications published prior to 2005 are excluded and only studies in English will be retrieved.

The searches will be conducted in the following databases:

- MEDLINE and MEDLINE in process (via OvidSP);

- PubMed;

- EMBASE (via OvidSP);

- PsycINFO (via OvidSP);
- NEXIS (media database useful for commercial/ market reports on over the counter sales of interventions such as gripe water);

- Database of Abstracts of Reviews of Effects (DARE) in the Cochrane Library;

- Health Technology Assessment Database (HTA Database) in the Cochrane Library;

- NHS Economic Evaluation Database (NHS EED) in the Cochrane Library;

- CEA Registry (via https://research.tufts-nemc.org/ cear4/);

- Evidence Search (https://www.evidence.nhs.uk/);

- OAISTER (http://www.oclc.org/oaister.en.html);

- RePEc (http://repec.org/).

The search strategy (figure 2) will be adapted suitably to perform efficiently in these databases.

The following conferences will be searched via Embase or via conference websites for the past 3 years, to identify recently completed research and to provide contact details, so that the authors can be contacted for full text:

- ISPOR;

- European Society for Paediatric Gastroenterology, Hepatology and Nutrition; 


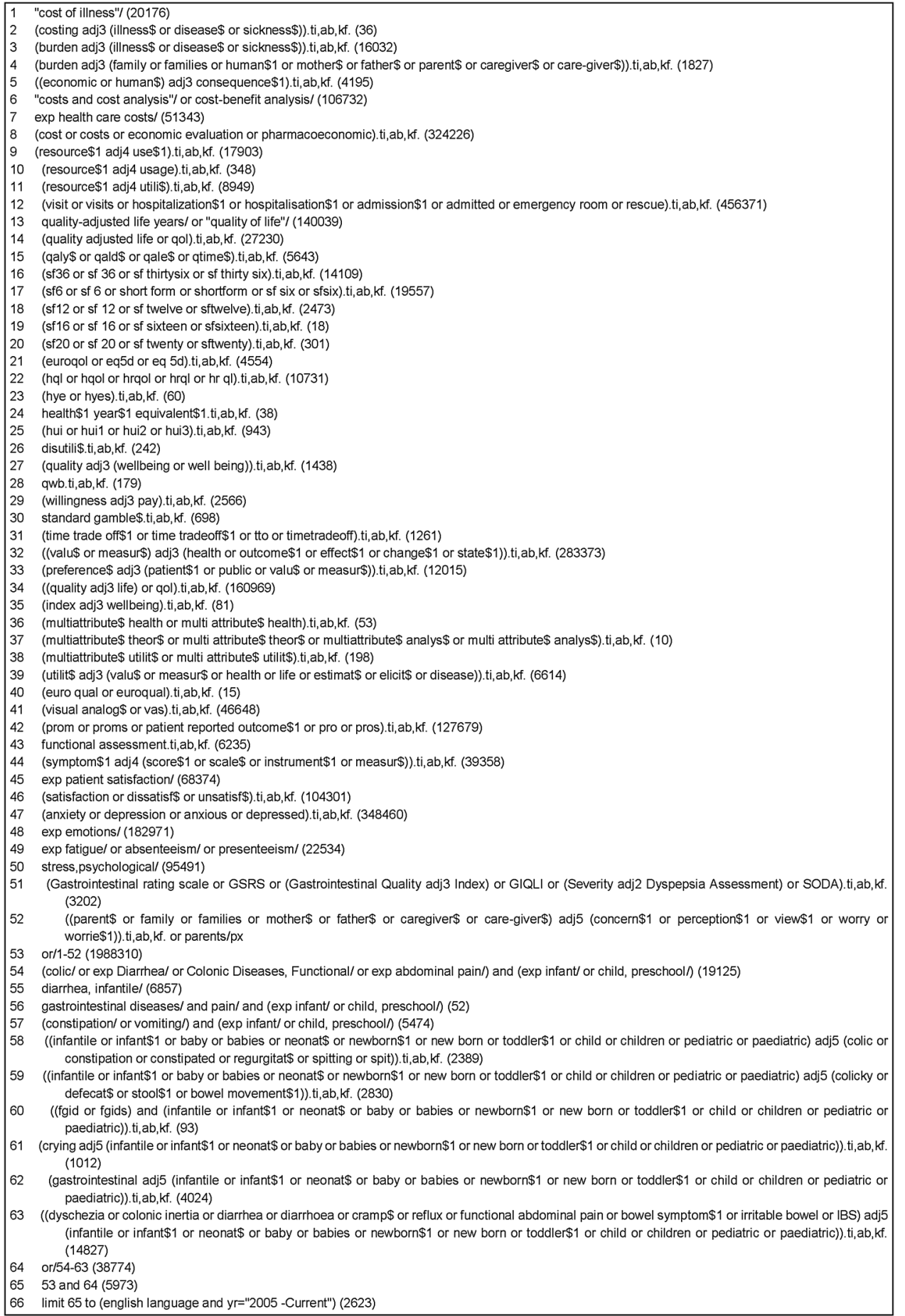

Figure 2 Search strategy for Ovid MEDLINE ${ }^{\circledR}$ in-process and other non-indexed citations and Ovid MEDLINE ${ }^{\circledR}<1946$ to present>.

- North American Society for Pediatric Gastroenterology, Hepatology and Nutrition;

- American Academy of Pediatrics National Conference;

- World Congress of Pediatric Gastroenterology, Hepatology and Nutrition.

The reference lists of any included studies and SRs will be checked to identify any studies that may have been missed by the database searches.

Details of all search strategies and search results will be provided in the SR report. The search and record selection process will be presented in a PRISMA flow diagram (http://www.prisma-statement.org/statement. htm).

Incidence/prevalence data would be required to perform a de novo calculation: these values will be sought from the published literature using a pragmatic focused epidemiology search in MEDLINE (figure 3). This strategy will be combined with specific country terms depending on the exemplar country. 
Figure 3 Pragmatic focused epidemiology search in MEDLINE.

1 (colic/ep or exp Diarrhea/ep or Colonic Diseases, Functional/ep or exp abdominal pain/ep) and (exp infant/ or child, preschool/)

2. (colic/ or exp Diarrhea/ or Colonic Diseases, Functional/ or exp abdominal pain/) and (exp infant/ or child, preschool/) and (incidence/ or prevalence/)

diarrhea, infantile/ep or (diarrhea, infantile/ and (incidence/ or prevalence/))

gastrointestinal diseases/ep and pain/ and (exp infant/ or child, preschool/)

gastrointestinal diseases/ and pain/ and (exp infant/ or child, preschool/) and (incidence/ or prevalence/)

(constipation/ep or vomiting/ep) and (exp infant/ or child, preschool/)

(constipation/ or vomiting/) and (exp infant/ or child, preschool/) and (incidence/ or prevalence/)

((infantile or infant\$1 or baby or babies or neonat $\$$ or newborn $\$ 1$ or new born or toddler $\$ 1$ or child or children or pediatric or paediatric) adj5 (colic or constipation or constipated or regurgitat\$ or spitting or spit)).ti,ab,kf. AND (incidence or prevalence or epidemiol\$).ti.

9 ((infantile or infant $\$ 1$ or baby or babies or neonat $\$$ or newborn $\$ 1$ or new born or toddler $\$ 1$ or child or children or pediatric or paediatric) adj5 (colicky or defecat\$ or stool\$1 or bowel movement\$1)).ti,ab,kf. AND (incidence or prevalence or epidemiol\$).ti.

10 ((fgid or fgids) and (infantile or infant\$1 or neonat $\$$ or baby or babies or newborn $\$ 1$ or new born or toddler $\$ 1$ or child or children or pediatric or paediatric)).ti,ab,kf. AND (incidence or prevalence or epidemiol\$).ti.

11 (crying adj5 (infantile or infant $\$ 1$ or neonat $\$$ or baby or babies or newborn $\$ 1$ or new born or toddler $\$ 1$ or child or children or pediatric or paediatric)).ti,ab,kf. AND (incidence or prevalence or epidemiol\$).ti.

12 (gastrointestinal adj5 (infantile or infant\$1 or neonat $\$$ or baby or babies or newborn $\$ 1$ or new born or toddler $\$ 1$ or child or children or pediatric or paediatric)).ti,ab,kf. AND (incidence or prevalence or epidemiol\$).ti.

13 ((Dyschezia or "Colonic Inertia" or diarrhea or diarrhoea or cramp\$ or reflux or "functional abdominal pain" or "bowel symptom\$1" or "irritable bowel" or IBS) adj5 (infantile or infant\$1 or neonat\$ or baby or babies or newborn $\$ 1$ or new born or toddler\$1 or child or children or pediatric or paediatric)).ti,ab,kf. AND (incidence or prevalence or epidemiol\$).ti.

or/1-13

\{country search terms\}

14 AND 15

\section{Study selection}

The results of the searches will be loaded into an EndNote library and deduplicated. Following the removal of obviously irrelevant records by a single experienced information specialist, studies will be added to Covidence software.(The Alfred Hospital, Monash University, National ICT Australia, et al. Covidence. Secondary Covidence [reviewing software]. https://www. covidence.org.)

Study selection will be undertaken by two reviewers independently, based first on information in the titles and abstracts (when available). Disagreements will be resolved by discussion or involvement of a third reviewer. Studies that are not excluded based on title and abstract screening will be retrieved as full-text documents. Records excluded based on an assessment of the full text will be recorded in an excluded studies table with a reason for exclusion.

\section{Data extraction}

Double independent reviewer data extraction will be undertaken into an Excel spreadsheet. Any disagreement between the two reviewers will be resolved by discussion and, if necessary, by a third reviewer.

The following data will be extracted from all eligible included publications:

- bibliographic data;

publication type;

- country;

- patient population (number, age, gender, ethnicity);

- definition of FGID and, if applicable, the definition of the symptoms related to FGID.

Topic specific information will also be extracted (table 2).

\section{Quality assessment}

Double independent reviewer quality assessment will be undertaken. Any disagreement between the two reviewers will be resolved by discussion and, if necessary, a third reviewer will be involved to adjudicate. The quality of COI studies will be assessed using the following questions:

- Was the type of COI method clearly described?

- Was the quality of the data used described?

- Were the data sources and dates reported clearly?

- Were data gaps described?

- Were data extrapolations reasonable?

- Were reasonable methods employed to avoid double counting?

- Were the calculations of cost clearly described?

- Were the methods used to handle uncertainty appropriate?

- Have the researchers offered assessments of the limitations of the study approach?

The quality of other studies will be assessed using instruments listed in table 3 .

\section{Data synthesis}

Studies identified for the SR and the extracted data will be summarised in tables.

Results will be presented by geographical region and/ or country and by specific FGID conditions. A distinction will be made in the tables to highlight interventions that are reported in the literature to be common or accepted practice and those that have been trialled.

An overview of treatments in use and costs incurred will be provided, with any geographical or other differences highlighted. An assessment of how the totals for 
Table 2 Data extraction elements

\begin{tabular}{|c|c|}
\hline $\begin{array}{l}\text { Systematic review } \\
\text { question }\end{array}$ & Data extraction \\
\hline $\begin{array}{l}\text { What are the current } \\
\text { treatment options } \\
\text { (medicinal and otherwise) } \\
\text { in use? }\end{array}$ & $\begin{array}{l}\text { Study aim and type } \\
\text { Treatment option } \\
\text { (description/dosage) } \\
\text { Prescription only/parent } \\
\text { purchase/fees } \\
\text { Timescales/length of } \\
\text { treatment } \\
\text { Number of treatments } \\
\text { Management of adverse } \\
\text { effects } \\
\text { Management of } \\
\text { comorbidities }\end{array}$ \\
\hline $\begin{array}{l}\text { What are the direct and } \\
\text { indirect costs for parents? }\end{array}$ & $\begin{array}{l}\text { Study aim and type } \\
\text { Burden components (eg, } \\
\text { over the counter } \\
\text { treatments, changes in } \\
\text { infant formula purchases } \\
\text { and the loss of income } \\
\text { through time taken off } \\
\text { work/inability to return to } \\
\text { work due to caring for the } \\
\text { infant or attend clinical } \\
\text { appointments) } \\
\text { Dates of data collection } \\
\text { Currency } \\
\text { The value of any costs } \\
\text { identified }\end{array}$ \\
\hline $\begin{array}{l}\text { What are the current } \\
\text { estimates of the COI of } \\
\text { FGIDs and related signs } \\
\text { and symptoms? }\end{array}$ & $\begin{array}{l}\text { Study aim and type } \\
\text { Definition of COI } \\
\text { Burden components (eg, } \\
\text { prescribed treatments, } \\
\text { visits to health } \\
\text { professionals) } \\
\text { Dates of data collection } \\
\text { Currency } \\
\text { Methods of data } \\
\text { identification/collection } \\
\text { Results: COI } \\
\text { Limitations }\end{array}$ \\
\hline
\end{tabular}

cost differ across countries and the elements that contribute to the differences-such as the difference in costs for treatments or the different ways healthcare is provided between countries-will be undertaken as far as the detail in the identified literature allows. If applicable, gaps and limitations of this approach will be highlighted and discussed in detail.

If data permit, one exemplar of what the COI would be in total across all cost areas considered for a developed country will be produced (de novo calculation) using data from the review where possible. Following data synthesis, a recommendation will be made regarding which country should be chosen, based on the completeness of evidence from the literature for making a COI calculation, and the accessibility of unpublished data to fill any gaps.
Table 3 Quality assessment instruments

\begin{tabular}{|c|c|}
\hline Study design & Tool \\
\hline RCTs & Cochrane Risk of Bias tool ${ }^{37}$ \\
\hline Economic & Drummond and Jefferson \\
\hline Evaluations & checklist $^{38}$ \\
\hline Case-control studies & $\begin{array}{l}\text { Case-control Checklist (CRD } \\
\text { report } 4)^{39} \text { Newcastle Ottawa } \\
\text { Scale }^{40}\end{array}$ \\
\hline Case series & $\begin{array}{l}\text { Case Series Checklist (CRD } \\
\text { report } 4)^{39}\end{array}$ \\
\hline Cohort studies & $\begin{array}{l}\text { Cohort Study Checklist (CRD } \\
\text { report } 4)^{39}\end{array}$ \\
\hline $\begin{array}{l}\text { Cross-sectional } \\
\text { studies }\end{array}$ & $\begin{array}{l}\text { Adapted Newcastle-Ottawa for } \\
\text { Cross-Sectional studies, adapted } \\
\text { by Herzog et al }(2013)^{41}\end{array}$ \\
\hline Decision models & Philips Checklist ${ }^{42}$ \\
\hline
\end{tabular}

All costs identified will be adjusted for inflation and converted to 2015 US\$.

\section{DISSEMINATION}

The high incidence of FGIDs and related signs and symptoms in infants has been reported and reviewed. ${ }^{6}{ }^{11}$ There is a gap in knowledge on the international, regional and national economic impact of infant FGIDs and related signs and symptoms which this SR seeks to close.

\section{CONCLUSION}

This SR will provide specific insight into the direct and indirect costs associated with FGIDs and related signs and symptoms to parents and third-party payers.

\section{Author affiliations}

${ }^{1}$ York Health Economics Consortium, University of York, York, UK

${ }^{2}$ Nutricia Research, Utrecht, The Netherlands

${ }^{3}$ Hospital Italiano, Buenos Aires, Argentina

${ }^{4}$ Pediatric Gastroentrology, Hepatology and Nutrition Division, Sheikh Khalifa Medical City, Abu Dhabi, United Arab Emirates

${ }^{5}$ Nationwide Children's Hospital, Columbus, Ohio, USA

${ }^{6}$ National University of Singapore, Singapore, Singapore

${ }^{7}$ University of Utrecht, Utrecht, The Netherlands

${ }^{8}$ Department of Paediatrics, Medical University of Warsaw, Warsaw, Poland

Contributors All authors gave input on the design and aim of the systematic review. HW, JG and TL designed the search strategy; HS, MM, SHQ, IL-W, MS and $C L$ gave input to the search strategy and the inclusion and exclusion criteria; JM and JG defined the data extraction elements; JM, JG, ME and TL wrote the protocol and this manuscript and its revisions; HS, MM, SHQ, IL-W, MS and CL revised the protocol and this manuscript.

Funding This work was carried out by York Health Economics Consortium, an independent consultancy, and was funded by Nutricia Research, Utrecht, The Netherlands. The protocol was developed by Julie Glanville and James Mahon.

Competing interests HS, MS, CL, MM and SHQ have served as consultants, advisory board members and/or speakers for companies manufacturing infant formulas, foods and probiotics or prebiotics. TL is an employee of Nutricia Research.

Ethics This is an SR of published studies and ethical committee approval is not required. The planned output for this SR is publication in a peer-reviewed journal. 
Provenance and peer review Not commissioned; externally peer reviewed.

Open Access This is an Open Access article distributed in accordance with the Creative Commons Attribution Non Commercial (CC BY-NC 4.0) license, which permits others to distribute, remix, adapt, build upon this work noncommercially, and license their derivative works on different terms, provided the original work is properly cited and the use is non-commercial. See: http:// creativecommons.org/licenses/by-nc/4.0/

\section{REFERENCES}

1. Brown M, Heine RG, Jordan B. Health and well-being in school-age children following persistent crying in infancy. $J$ Paediatr Child Health 2009:45:254-62.

2. Rautava $\mathrm{P}$, Lehtonen L, Helenius $\mathrm{H}$, et al. Infantile colic: child and family three years later. Pediatrics 1995;96(Pt 1):43-7.

3. Vik T, Grote V, Escribano J, et al. Infantile colic, prolonged crying and maternal postnatal depression. Acta Paediatr 2009;98:1344-8.

4. Miller-Loncar C, Bigsby R, High P, et al. Infant colic and feeding difficulties. Arch Dis Child 2004;89:908-12.

5. Indrio F, Di Mauro A, Riezzo G, et al. Infantile colic, regurgitation, and constipation: an early traumatic insult in the development of functional gastrointestinal disorders in children? Eur J Pediatr 2015;174:841-2.

6. Vandenplas $\mathrm{Y}$, Abkari A, Bellaiche $\mathrm{M}$, et al. Prevalence and health outcomes of functional gastrointestinal symptoms in infants from birth to 12 months of age. J Pediatr Gastroenterol Nutr 2015:61:531-7.

7. Mitchell CM, Drossman DA. Survey of the AGA membership relating to patients with functional gastrointestinal disorders. Gastroenterology 1987;92(Pt 1):1282-4.

8. Drossman DA. The functional gastrointestinal disorders and the Rome III process. Gastroenterology 2006;130:1377-90.

9. Hyman PE, Milla PJ, Benninga MA, et al. Childhood functional gastrointestinal disorders: neonate/toddler. Gastroenterology 2006;130:1519-26.

10. van Tilburg MA, Hyman PE, Walker L, et al. Prevalence of functional gastrointestinal disorders in infants and toddlers. $J$ Pediatr 2015;166:684-9.

11. Iacono G, Merolla R, D'Amico D, et al. Gastrointestinal symptoms in infancy: a population-based prospective study. Dig Liver Dis 2005;37:432-8.

12. Shamir R, St James-Roberts I, Di Lorenzo C, et al. Infant crying, colic, and gastrointestinal discomfort in early childhood: a review of the evidence and most plausible mechanisms. J Pediatr Gastroenterol Nutr 2013;57(Suppl 1):S1-45.

13. Lucassen P. Colic in infants. BMJ Clin Evid 2015;2015:pii:0309.

14. Garrison MM, Christakis DA. A systematic review of treatments for infant colic. Pediatrics 2000;106(Pt 2):184-90.

15. Drug and Therapeutics Bulletin. Management of infantile colic. BMJ 2013;347:f4102.

16. Harb T, Matsuyama M, David M, et al. Infant Colic-what works: a systematic review of interventions for breast-fed infants. J Pediatr Gastroenterol Nutr 2016;62:668-86.

17. Hall B, Chesters J, Robinson A. Infantile colic: a systematic review of medical and conventional therapies. J Paediatr Child Health 2012;48:128-37.

18. Vandenplas $\mathrm{Y}$, Alarcon $\mathrm{P}$, Alliet $\mathrm{P}$, et al. Algorithms for managing infant constipation, colic, regurgitation and cow's milk allergy in formula-fed infants. Acta Paediatr 2015;104:449-57.

19. Vandenplas $Y$, Gutierrez-Castrellon $P$, Velasco-Benitez $C$, et al. Practical algorithms for managing common gastrointestinal symptoms in infants. Nutrition 2013;29:184-94.

20. Vandenplas Y, Rudolph CD, Di Lorenzo C, et al. Pediatric gastroesophageal reflux clinical practice guidelines: joint recommendations of the North American Society for Pediatric Gastroenterology, Hepatology, and Nutrition (NASPGHAN) and the European Society for Pediatric Gastroenterology, Hepatology, and
Nutrition (ESPGHAN). J Pediatr Gastroenterol Nutr 2009;49:498-547.

21. Lightdale JR, Gremse DA, Section on Gastroenterology Hepatology, and Nutrition. Gastroesophageal reflux: management guidance for the pediatrician. Pediatrics 2013;131:e1684-95.

22. Gieruszczak-Białek D, Konarska Z, Skórka A, et al. No effect of proton pump inhibitors on crying and irritability in infants: systematic review of randomized controlled trials. J Pediatr 2015;166:767-70 e3.

23. Vandenplas Y. Management of paediatric GERD. Nat Rev Gastroenterol Hepatol 2014;11:147-57.

24. Czinn SJ, Blanchard S. Gastroesophageal reflux disease in neonates and infants: when and how to treat. Paediatr Drugs 2013;15:19-27.

25. Lucassen PL, Assendelft WJ, van Eijk JT, et al. Systematic review of the occurrence of infantile colic in the community. Arch Dis Child 2001;84:398-403.

26. Quitadamo P, Miele E, Alongi A, et al. Italian survey on general pediatricians' approach to children with gastroesophageal reflux symptoms. Eur J Pediatr 2015;174:91-6.

27. Headley J, Northstone K. Medication administered to children from 0 to 7.5 years in the Avon Longitudinal Study of Parents and Children (ALSPAC). Eur J Clin Pharmacol 2007;63:189-95.

28. Lucassen P. Colic in infants. BMJ Clin Evid 2010;2010:pii:0309.

29. Illingworth C, Timmins J. Gripe water: what is it? Why is it given? Health Visit 1990;63:378.

30. Chinawa JM, Ubesie AC, Adimora GN, et al. Mothers' perception and management of abdominal colic in infants in Enugu, Nigeria. Niger J Clin Pract 2013;16:169-73.

31. Sas D, Enrione MA, Schwartz RH. Pseudomonas aeruginosa septic shock secondary to 'gripe water' ingestion. Pediatr Infect Dis $J$ 2004;23:176-7.

32. Snyder J, Brown P. Complementary and alternative medicine in children: an analysis of the recent literature. Curr Opin Pediatr 2012;24:539-46.

33. Kheir AE. Infantile colic, facts and fiction. Ital J Pediatr 2012;38:34.

34. Barr RG. The normal crying curve: what do we really know? Dev Med Child Neurol 1990;32:356-62.

35. Morris S, James-Roberts IS, Sleep J, et al. Economic evaluation of strategies for managing crying and sleeping problems. Arch Dis Child 2001:84:15-19.

36. Sommers T, Corban C, Sengupta N, et al. Emergency department burden of constipation in the United States from 2006 to 2011. Am J Gastroenterol 2015;110:572-9.

37. Higgins JPT, Altman DG, Sterne JAC. Assessing risk of bias in included studies. In: Higgins JPT, Green S, eds. Cochrane Handbook for Systematic Reviews of Interventions, 2011.

38. Drummond MF, Jefferson TO. Guidelines for authors and peer reviewers of economic submissions to the BMJ. The BMJ economic evaluation working party. BMJ 1996;313:275-83.

39. Centre for Reviews and Dissemination. Undertaking systematic reviews of research on effectiveness: CRD's guidance for those carrying out or commissioning reviews. Report 4. 2nd edn. York: University of York, 2001.

40. Wells GA, Shea B, O'Connell D, et al. The Newcastle-Ottawa Scale (NOS) for assessing the quality of nonrandomised studies in meta-analyses. Secondary The Newcastle-Ottawa Scale (NOS) for assessing the quality of nonrandomised studies in meta-analyses [webpage]. 2014. http://www.ohri.ca/programs/clinical_epidemiology/ oxford.asp

41. Herzog R, Álvarez-Pasquin MJ, Díaz C, et al. Are healthcare workers' intentions to vaccinate related to their knowledge, beliefs and attitudes? A systematic review. BMC Public Health 2013; 13:154.

42. Philips Z, Ginnelly L, Sculpher M, et al. Review of guidelines for good practice in decision-analytic modelling in health technology assessment. Health Technol Assess 2004;8:iii-iv, ix-xi, 1-158. http://www.journalslibrary.nihr.ac.uk/ data/assets/pdf file/0015/ 65121/FullReport-hta8360.pdf 
Correction: Costs associated with functional gastrointestinal disorders and related signs and symptoms in infants: a systematic review protocol

Glanville J, Ludwig T, Lifschitz C, et al. Costs associated with functional gastrointestinal disorders and related signs and symptoms in infants: a systematic review protocol. BMJ Open 2016;6:e11475. The name segmentation of the 7th author is incorrect. The author's surname is Quak; first names are Seng Hock. This author should be cited as 'Quak SH'.

Open Access This is an Open Access article distributed in accordance with the Creative Commons Attribution Non Commercial (CC BY-NC 4.0) license, which permits others to distribute, remix, adapt, build upon this work non-commercially, and license their derivative works on different terms, provided the original work is properly cited and the use is non-commercial. See: http://creativecommons.org/licenses/by-nc/4.0/

BMJ Open 2016;6:e011475corr1. doi:10.1136/bmjopen-2016-011475corr1 